\title{
Gastrointestinal Stromal Tumors: Whether Mutation Guided Diagnosis Matter
}

\author{
Gayatri $\mathbf{G}^{1^{*}}$, Borgohain $\mathbf{M}^{2}$, Das $\mathbf{G}^{3}$, Changsan $\mathrm{LL}^{4}$, Kouli $\mathbf{R}^{5}$, Manta $\mathrm{A}^{6}$ and Teronpi $\mathrm{J}^{7}$ \\ ${ }^{1}$ Assistant Professor, Department of Pathology, Assam Medical College, Dibrugarh, Assam, India \\ ${ }^{2}$ Professor, Department of Pathology, Assam Medical College, Dibrugarh, Assam, India \\ ${ }^{3}$ Assistant Professor, Department of General Surgery, Assam Medical College, Dibrugarh, Assam, India \\ ${ }^{4}$ Post Graduate trainee, Department of Pathology, Assam Medical College, Dibrugarh, Assam, India \\ ${ }^{5}$ Associate Professor, Department of Pathology, Assam Medical College, Dibrugarh, Assam, India \\ ${ }^{6}$ Demonstrator of Pathology, Department of Pathology, Assam Medical College, Dibrugarh, Assam, India \\ ${ }^{7}$ Post Graduate trainee, Department of Pathology, Assam Medical College, Dibrugarh, Assam, India
}

*Corresponding author: Gyatri G, Assistant Professor, Department of Pathology, Assam Medical College, Dibrugarh, Assam, India, Tel: +91-9435030084; E-mail: gayatrigogoi303@gmail.com

Received date: June 27, 2016; Accepted date: February 25, 2017; Published date: March 4, 2017

Copyright: (c) 2017 Gaytri G. This is an open-access article distributed under the terms of the Creative Commons Attribution License, which permits unrestricted use, distribution, and reproduction in any medium, provided the original author and source are credited.

\begin{abstract}
Introduction: Gastrointestinal Stromal Tumor (GIST), now the most common mesenchymal tumor of the Gastrointestinal Tract (GIT), spans a clinical spectrum from benign to malignant. It has been frequently studied, especially with regard to its successful targeted therapy using imatinib mesylate. Approximately $70-80 \%$ of GISTs have gain of function mutation of the KIT gene. The aim of the study is to describe spectrum of clinical presentation and histomorphologic characteristics in classic GIST with CD117 immunostaining in a tertiary care hospital from north east India. Design: This is a descriptive cross sectional study of cases encountered over one-year period. Cases included after histomorphology and immunohistochemistry staining with CD117.
\end{abstract}

Results: A total of 10 cases of GIST like histomorphology were studied and 6 were confirmed as GIST and included in the study. Four were males and two females with age ranged from 32 to 55 years. The cases had variable clinical presentations with abdominal pain and lump, melena and bleeding per rectum. Two of the cases were located in the stomach, one in the duodenum involving the periampullary area, one each in the ileocaecal region, transverse colon and rectum. Most of the cases grossly presented as a polypoidal growth. Four of the cases showed typical spindle cell morphology composed of interlacing bundles of uniform spindle shaped cells. One had a mixed morphology with features of both spindle cell and epithelioid pattern and one case mostly epithelioid type. Two cases showed intense immunoreactivity for CD117 in $90 \%$ of the tumour cells, three cases showed immunoreactivity for CD117 in $70-80 \%$ and in one case $50 \%$. The management by surgical resections followed by 6 months imatinib mesylate treatment of 5 cases in the study had been showing good response for last two years.

Conclusion: The molecular phenotype is an important consideration in the treatment of patients from prognostic point of view. Those with mutations in KIT or PDGFRA often respond to the tyrosine kinase inhibitor imatinib in contrast, tumors without these mutations are generally resistant. So a uniform category of cases with cKIT mutation may be helpful to study treatment outcome besides standard predictor of biologic behavior. It would be interesting to see whether mutation guided diagnosis has any bigger role in prognosis of classic GIST in future studies involving larger sample size.

Keywords: GIST; c-KIT mutation; CD117; Imatinib mesylate

\section{Aim and Objective}

The study is to describe spectrum of clinical presentation and histomorphologic characteristics in classic GIST with CD117 immunostaining in a tertiary care hospital from north east India.

\section{Introduction}

Gastrointestinal stromal tumor GIST is the most common primary mesenchymal tumour of the gastrointestinal tract and spans a clinical spectrum from benign to malignant [1]. Two-thirds of GIST arise from the stomach, and one-fourth arises in the small intestine. Colorectal lesions account for approximately $10 \%$ of GISTs [2]. Although, originally classified as smooth muscle tumours, GISTs are now known to arise from interstitial cells of Cajal or precursors of such cells [3]. Approximately $70-80 \%$ of GISTs has gain of function mutation of the KIT gene and is immunopositive for CD117. Most of the remaining 5\% of GISTs have mutation of the platelet-derived growth factor alpha (PDGFRA) gene [2]. CD117 (KIT) expression (a proto-oncogene protein) has emerged as a sensitive, although not entirely, specific marker, being positive in $95 \%$ of the cases. GIST has an annual, worldwide incidence of approximately 1.5 per 100,000 persons. It occurs most frequently in adults aged 50-70 years and has an equal gender distribution [4]. Although they comprise less than $1 \%$ of all gastrointestinal tumors, GIST are the most common mesenchymal 
tumors of the GI tract. The incidence of GIST is reported as low (i.e., 2 in $1,00,000)$ in India.

\section{Materials and Methods}

A cross sectional study was carried out in a tertiary care teaching hospital in Northeast India over a period of 1 year duration. All clinical details of cases were recorded who were histologically showing suspicion of GIST or differential diagnosis. The standard protocol of gastro intestinal tumour grossing were maintained, where site, size, tumour margins, other features including presence of regional nodes were examined. Then representative tissue sections were taken and processed according to standard operating procedure of histopathology tissue processing. Formalin fixed, paraffin embedded blocks was prepared and 3-5 mm thickness sectioned slides were stained with $\mathrm{H} \& \mathrm{E}$ stain. Then they were viewed under light microscope in all the cases. All histologically suspected as GIST was studied by further by Immunohistochemistry (IHC) using appropriate antibodies. A thickness of $3-4 \mathrm{~mm}$ thin sections were taken in 3 aminopropyl triethoxysilane coated slides both from tests blocks and control blocks for IHC procedure. Antigen retrieval for target epitopes were carried out in dedicated micro oven antigen retrieval system with uniform heating and controlled temperatures. The panel of antibodies included were CD117, SMA Desmin, vimentin, S-100, ki-67. Only IHC confirming diagnoses of GIST by positive staining in CD117 were included in results and correlations were carried out in the study.

\section{Result and Interpretation}

\section{Case 1}

A 40 years old, female patient presented with vague pain in the abdomen and bleeding per rectum. Colonoscopy showed a broad based polypoidal lesion in transverse colon and USG showed hyper echoic polypoidal lesion in transverse colon. All other investigations were normal except peripheral blood picture showed normochromic normocytic (Figure 1) anemia. Patient underwent a right sided hemicolectomy with an ileocolic anastomosis and the specimen was sent for histopathological examination. Grossly, a tumor mass of size 5 $\times 6(\mathrm{~cm} \times \mathrm{cm})$ was seen with gray pink, solid in cut section and areas of hemorrhage and necrosis.

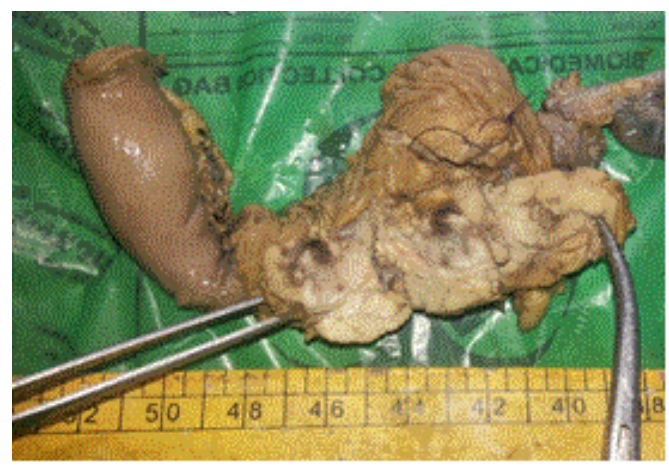

Figure 1: Gross picture of GIST involving the duodenum.

\section{Case 2}

A 55-years-old male presented to surgery outpatient department with abdominal pain. On palpation there was abdominal mass. Medical history and family history were unremarkable. Upper gastrointestinal endoscopy reveal greater (Figure 2) curvature ulcerated growth without active bleeding; $4-5 \mathrm{~cm}$ in size polypoidal in nature with overlying multiple ulcerations and sloughing [Endo figure].

It was initially diagnosed as hyperplastic polyp. The patient underwent partial gastrectomy with loop gastrojejunostomy. Gross specimen showed both exophytic $(7 \times 7 \mathrm{~cm})$ and endophytic growth $(4$ $\times 4 \mathrm{~cm})$ in the stomach wall which had areas of hemorrhage and necrosis.

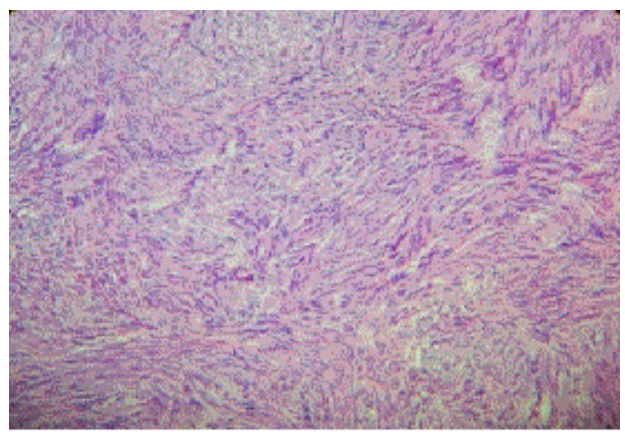

Figure 2: GIST composed of interlacing fascicles of cells with cigarshaped nuclei, low power view.

\section{Case 3}

A 55 years old lady presented with abdominal swelling and pain since 8 days. There was history of jaundice and malaena too after admission. CT scan abdomen showed features of heterogeneously enhancing wall thickening of the distal common (Figure 3) bile duct causing its luminal narrowing leading to biliary and pancreatic ductal prominence, infiltrating the second part of the duodenum. MRCP reveals ill-defined soft tissue lesion in the region of pancreatic head causing widening of the C-loop and suspected involvement of wall of second part of duodenum. The patient underwent Whipple's procedure pancreaticoduodenectomy. Gross specimen showed solid growth $(6 \times$ $5 \mathrm{~cm}$ ) with areas of hemorrhage and necrosis involving the periampullary region and second part of duodenum. 
Citation: Gayatri G, Borgohain M, Das G, Changsan LL, Kouli R, Manta A and Teronpi J (2017) Gastrointestinal Stromal Tumors: Whether Mutation Guided Diagnosis Matter. Arch Surg Oncol 3: 121. doi:10.4172/2471-2671.1000121

Page 3 of 5

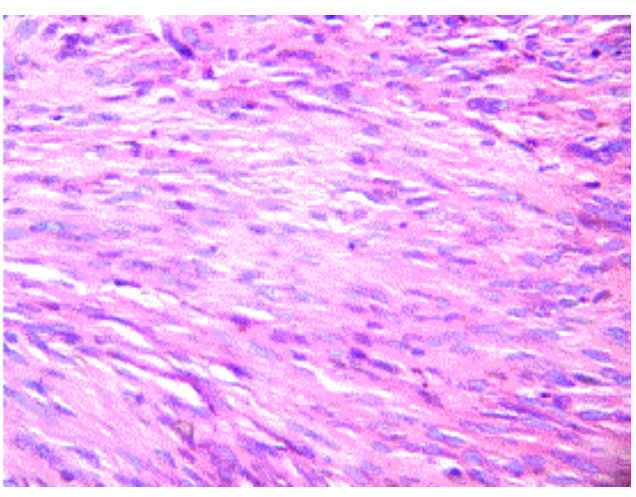

Figure 3: GIST in high power view with typical spindled cell morphology.

\section{Case 4}

A 48 years old male presented with passage of black stool. Upper GI endoscopy showed 4-5 $\mathrm{cm}$ polypoidal ulcerated growth with in greater curvature of the stomach. Small biopsied specimen was sent for histopathological examination (Figure 4) and later on surgical excision of the mass was done on confirmation as a case of GIST.

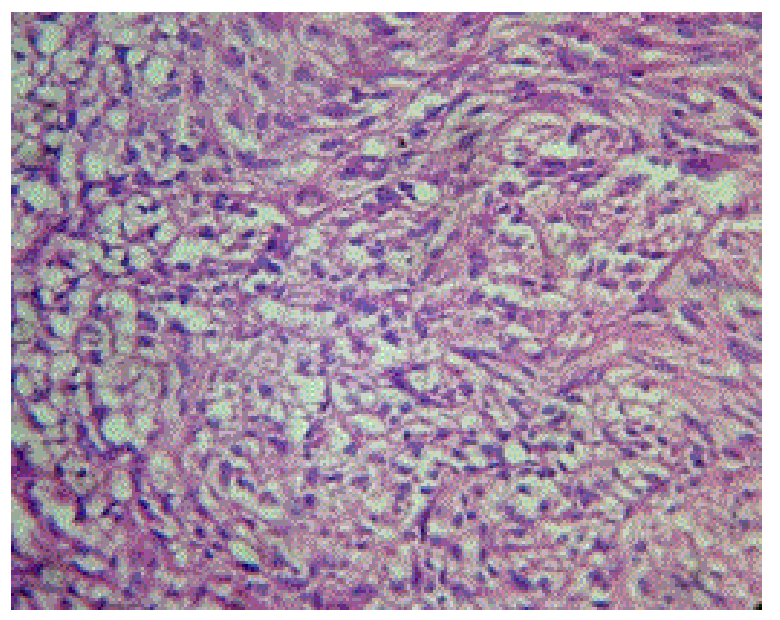

Figure 4: Epithelioid GIST of the stomach with rounded nuclei and a clear cytoplasm.

\section{Case 5}

A 46 years old male had bleeding per rectum. CECT abdomenGiant hyperplastic rectal polyp/polypoidal intraluminal rectal neoplasia. Small biopsied (Figure 5) specimen was sent for histopathological examination which histomorphologically resemble Schwannoma which turned out GIST on IHC confirmation.

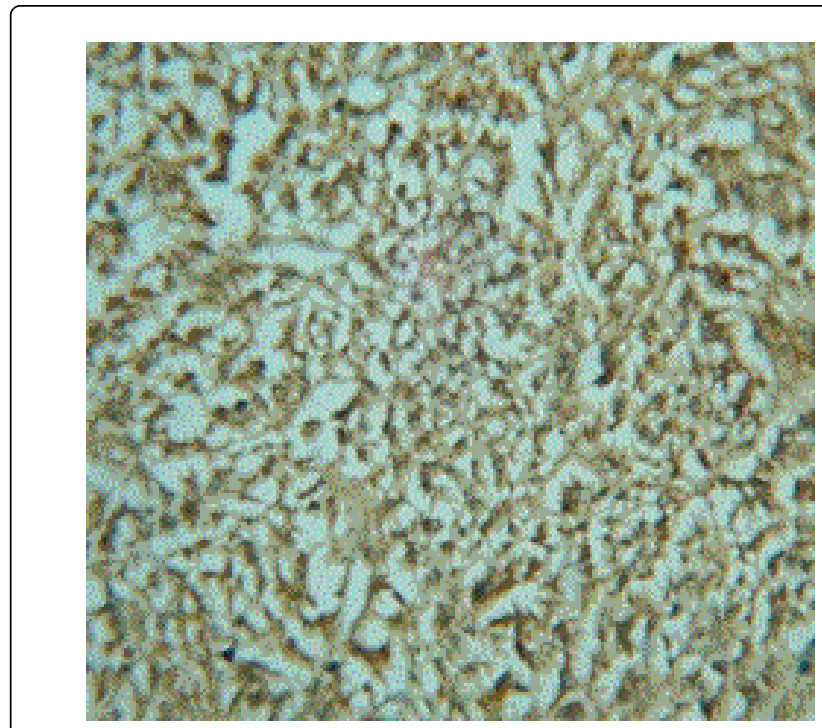

Figure 5: CD117 immunostaining, 40X.

\section{Case 6}

A 55 years old male presented with abdominal swelling since 3 months. Exploratory laparotomy revealed a mass originating from ileocaecal region.

Right sided extended hemicolectomy with (Figures 6 and 7) end to side ileocolic anastomosis done. Excision biopsy and immunohistochemistry confirmed GIST. Postoperative period was uneventful.

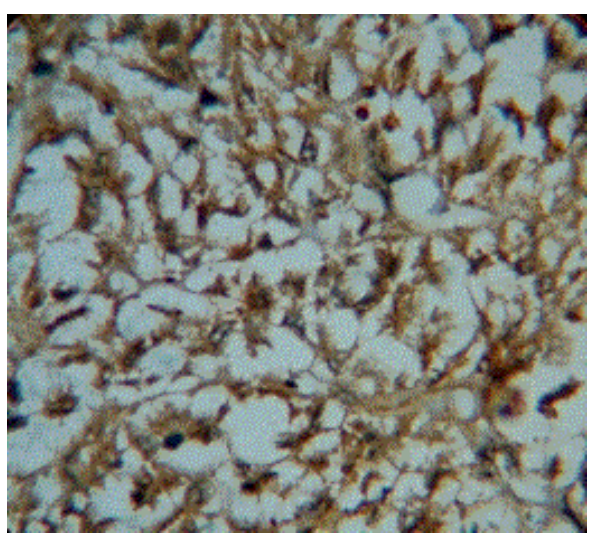

Figure 6: CD117 staining, 100X. 
Page 4 of 5

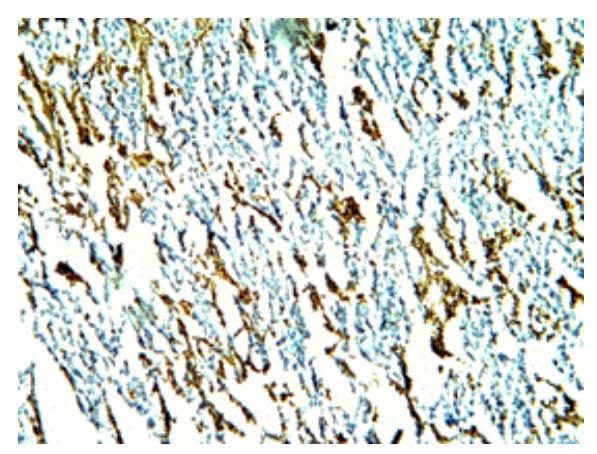

Figure 7: Vimentin immunoreactivity in tumor cells.

\section{Results}

A total of 10 GIST like histological cases were studied and 6 cases were confirmed as GIST and included in the study. Of the 6 cases, four were males and two females. Age group of the patients ranged from 32-55 years (Table 1 ).

\begin{tabular}{|l|l|l|l|l|}
\hline Cases & Age & Sex & Clinical presentation & Sites \\
\hline
\end{tabular}

\begin{tabular}{|l|l|l|l|l|}
\hline 1. & 40 & $\mathrm{~F}$ & $\begin{array}{l}\text { Vague abdominal pain and } \\
\text { bleeding per rectum }\end{array}$ & Transverse colon \\
\hline 2. & 55 & $\mathrm{M}$ & Abdominal pain & Stomach \\
\hline 3. & 55 & $\mathrm{~F}$ & Abdominal swelling and pain & $\begin{array}{l}\text { Duodenum and } \\
\text { periampullary part }\end{array}$ \\
\hline 4. & 48 & $\mathrm{M}$ & $\begin{array}{l}\text { Abdominal pain and passage of } \\
\text { black stool }\end{array}$ & Stomach \\
\hline 5. & 46 & $\mathrm{M}$ & Bleeding per rectum & Rectum \\
\hline 6. & 32 & $\mathrm{M}$ & Pelvic swelling & lleocaecal region \\
\hline
\end{tabular}

Table 1: Clinical details of the 6 cases.

The cases had variable clinical presentations with abdominal pain and lump, malaena and bleeding per rectum. Two of the cases were located in the stomach, one in the duodenum involving the periampullary area, one each ileocaecal region, transverse colon and rectum. Most of the cases grossly presented as a polypoidal growth. Cut sections revealed gray to pink, circumscribed firm to solid growth with occasional areas of hemorrhages and necrosis. The sizes ranges from 4.5-7 cm in case of completely resected tumour. Histology revealed variable pictures (Table 2).

\begin{tabular}{|c|c|c|c|c|c|c|}
\hline Cases & Size of the tumor in $\mathrm{cm}$ & Histomorphology & $\begin{array}{l}\text { Mitotic } \\
\text { count }\end{array}$ & $\begin{array}{l}\text { Histological } \\
\text { diagnosis }\end{array}$ & CD117 & $\begin{array}{l}\text { Risk of progression to } \\
\text { malignacy }\end{array}$ \\
\hline 1. & $7 \times 7 / 4 \times 4$ & $\begin{array}{l}\text { Both spindle and } \\
\text { epithelioid pattern }\end{array}$ & $>10$ & GIST & + & high \\
\hline 2. & $4 \times 5$ & Spindled & 4 & GIST & + & Low \\
\hline 3. & $6 \times 5$ & Spindle & 10 & GIST & + & high \\
\hline 4. & $3 \times 4$ & Epitheioid & 3 & GIST & + & low \\
\hline 5. & $0.5 \times 0.5$ & $\begin{array}{l}\text { Spindle with Nuclear } \\
\text { palisading }\end{array}$ & 4 & GIST & + & low \\
\hline 6. & $6 \times 0.5$ & Spindle & 1 & GIST & + & low \\
\hline
\end{tabular}

Table 2: Histomorphologic and prognostic characteristics.

Three of the cases showed typical spindle cell morphology composed of interlacing bundles of uniform spindle shaped cells with ovoid to elongated blunt ended nuclei and fibrillary eosinophilic cytoplasm as can be seen in Figures 2 and 3. Focal areas of hemorrhage and necrosis were present. One of the cases had a mixed morphology of both spindle cell and epithelioid pattern. Two cases showed intense immunoreactivity for CD117 in $90 \%$ of the tumour cells, three cases showed immunoreactivity for CD117 in $70-80 \%$ and in one case $50 \%$. All the cases were positive for Vimentin, S100 was focally positive in one. Others markers like Desmin, CK were negative in all the cases.

\section{Discussion}

GIST typically occurs in older adults with peak incidence between the fifth and eight decades of life and same sex preponderance. In the present study, age group ranged from 32 to 55 years which is slightly lower than other studies. The most common presenting symptom was abdominal pain. Four cases in the study had spindle cell morphology; one case had histomorphological features of mixed spindle cell and epithelioid variant while another case was pure epithelioid type. In a study by Lakshmi et al. [5], $66 \%$ of the tumors had spindle cell morphology, $24 \%$ of the tumors had mixed spindle and epithelioid cell morphology, $27 \%$ of the tumors had nuclear palisading while 4 cases had pure epithelioid cell morphology. The histologically epithelioid pattern found in our study posed diagnostic dilemma because of its histomorphological resemblance to mucinous carcinoma. The epithelioid cells are large polyhedral shaped with abundant pale or clear cytoplasm giving a picture of epithelial origin carcinoma One case showed spindle cells with nuclear palisading which led was initially diagnosed as Schwannoma. Subsequently, on doing immunohistochemistry, it was found to be strongly immune-reactive for CD117, and hence was confirmed as GIST. GISTs demonstrate variable histology, including sheet-like arrangement, short fascicles, whorls, storiform, and organoid patterns which lead to differential diagnosis of other soft tissue tumour such as leiomyoma, schwannoma, fibroma etc. 
Mitotic activity was assessed to stratify tumours into risk groups as described by Fletcher et al. [6]. Tumors less than $2 \mathrm{~cm}$ in size with a mitotic count $<5 / 50$ in a high power field (HPF) are categorized as very low risk; tumors between $2-5 \mathrm{~cm}$ with a mitotic count $<5 / 50 \mathrm{HPF}$ are categorized as low risk; tumors $<5 \mathrm{~cm}$ in size with a mitotic count of 6-10/50 HPF or tumors with a size of $5-10 \mathrm{~cm}$ and a mitotic count of $<5 / 50 \mathrm{HPF}$ are categorized as intermediate risk; and tumors $>5 \mathrm{~cm}$ with a mitotic count of $>5 / 50 \mathrm{HPF}$, tumors greater than $10 \mathrm{~cm}$ and any mitotic rate, and tumors of any size with a mitotic rate $>10 / 50 \mathrm{HPF}$ are classified as high risk [6]. In the present study, as per tumour size and mitotic activity, 2 cases were in High risk category and 4 cases were in Low risk category.

The most important immunohistochemical marker of GIST is expression of KIT (CD117), which is found in the vast majority of GIST. Twenty cases at entry to the study were classified as smooth muscle tumors until CD117-positive immunostaining proved them to be GIST, emphasizing that CD117 immunochemistry is mandatory to confirm the diagnosis of GIST, even on small biopsy specimens [7]. In the present study, on doing immunohistochemistry, two cases showed intense immunoreactivity for CD117 in 90\% of the tumour cells, three cases showed immunoreactivity for CD117 in $70-80 \%$ of tumour cells; one case stain $\mathrm{CD} 117$ is $50 \%$ while one case $\mathrm{CD} 117$ stain was repeated [8]. Staining is usually strong and widespread and may be distributed diffusely in the cytoplasm, localized to a perinuclear dot, or membranous. However in some cases it can be patchy or weak. PDGFRA genotype can help in CD117 negative cases, but was not performed in our study which would be a limitation in our study we have included only CD117 positive cases in a study by Rubin et al. [8]. immunohistochemical analyses revealed strong and diffuse KIT (CD117) expression in each of the 48 cases of GISTs. Also Lakshmi et al., found 87 of the 92 cases $(94.5 \%)$ to be immunoreactivity for CD117.

The criterion focused in our study was exclusively based on CD117 immunostaining diagnosis of GISTs and all other GIST looking tumours were excluded. The management of high risked category cases was done with surgical resections followed by 6 months imatinib mesylate treatment and low risked category cases were surgically resected. We also observed good response in all the cases except in one case with rectal GISTs who could not be followed up. GISTs are resistant to conventional chemotherapy and radiation. Surgical resection is still the best modality of treatment. The survival of patients with metastatic and inoperable GIST has improved dramatically since the introduction of imatinib mesylate into treatment protocols [6]. The finding that most GISTs have KIT expression has led to the hypothesis that targeting KIT protein and selectively inhibiting KIT receptor tyrosine kinase might be useful in medical therapy in addition to it being a diagnostic marker for GIST according to Fletcher et al. [6].

\section{Conclusion}

GIST is the most common mesenchymal tumours of the GI system. The primary treatment consists of surgical excision of the tumor with a good margin of normal tissue. Though the molecular phenotype is an important consideration in the treatment of patients from a prognostic point of view, in a resource limited setting $\mathrm{CD} 117$ is taken as major criteria for diagnosis of GIST.

\section{References}

1. Miettinen M, Fletcher CDM, Kindblom LG, Tsui WMS (2010) Mesenchymal tumours of the stomach, WHO classification of tumours of the digestive system (4th edn.).

2. Mills S, Greenson J, Hornick J, Longacre T, Reuter V (2015) STERNBERG'S Diagnostic Surgical Pathology (6th edn.). China: Wolters Kluwer Health.

3. Hornick LJ (2007) Tumors of the small and large intestines, including anal canal. In Fletcher CD Diagnostic histopathology of tumors (4th edn). Philadelphia, p: 437.

4. Corless C (2004) Biology of gastrointestinal stromal tumors. J Clin Oncol 22: 3813-3825.

5. Lakshmi VA, Chacko RT, Kurian S (2010) Gastrointestinal stromal tumors: A 7-year experience from a tertiary care hospital. Indian J Pathol Microbiol 53: 628-633.

6. Fletcher CD, Berman JJ, Corless C, Gorstein F, Lasota, J et al. (2002) Diagnosis of gastrointestinal tumors: a consensus approach. Int J Surg Pathol 10: 81-89.

7. Campbell F, Lauwers GY (2006) Tumors of the esophagus and stomach (4th edn.). Elsevier.

8. Rubin BR, Singer S, Tsao C, Duensing A, Lux ML, et al. (2001) KIT activation is a ubiquitous feature of gastrointestinal stromal tumors. Cancer Res 61: 8118-8121. 